\title{
Evaluation of the consistency of fiber reinforced cementitious composites
}

\author{
Josef P. Kaufmann • Frank Winnefeld • \\ Daniela Hesselbarth · Walter Trindler
}

Received: 29 June 2005 / Accepted: 26 July 2005

(C) RILEM 2006

\begin{abstract}
The rheological properties of fresh concrete, mortar or cement paste are among the most important parameters when cementitious building materials are placed. New material designs, like high or ultrahigh performance concretes, include the addition of a high volume of fibers to the fresh mix influencing its workability properties. However, the analysis of the rheological properties of fiber reinforced cementitious composites is difficult. Conventional methods mostly do not apply, especially when a high fiber content and relatively stiff mixtures are used. For this reason, a new method was developed to evaluate the workability of fiber reinforced composites. This method was applied to carbon and PVA fiber reinforced high performance composites and was used to optimize the rheological properties of these composites for an application in a centrifugation casting process.
\end{abstract}

Résumé Les propriétés rhéologiques de la pâte de ciment, du mortier et du béton frais font parties des paramètres les plus importants pour la mise en place des matériaux cimentaires. De nouveaux concepts de formulation, tels que les béton hautes ou très hautes performances, comprennent une adjonction d'un grand volume de fibres qui influence l'ouvrabilité. L'analyse

J.P. Kaufmann · F. Winnefeld · D. Hesselbarth · W. Trindler

EMPA, Swiss Laboratories for Materials Testing and Research, Laboratory for Concrete \& Construction Chemistry, Dübendorf (Switzerland) des propriétés rhéologiques de tels systèmes est difficile. Les méthodes conventionnelles ne sont pas applicables, surtout quand les composites sont peu fluides à cause de leur teneur élevée en fibres. C'est la raison pour laquelle une nouvelle méthode a été développée pour évaluer l'ouvrabilité des composites renforcés de fibres. Cette méthode a été appliquée pour optimiser les propriétés rhéologiques de composites hautes performances renforcés de fibres de carbone et d'alcool de polyvinyle pour un procédé de fabrication par centrifugation.

\section{Introduction}

The consistency or workability of the fresh mixture is an essential property of mortar and concrete, an overview is given for example in $[1,2]$. With the development of self compacting or high performance concretes the rheological properties became even more important. They are determined in many different ways as shown by [3]. Some methods are based on the spread of the freshly mixed material on a table when it is filled into a cone that is lifted afterwards (EN 1015-3, EN 12350-3 etc.). Others are based on the measurement of the time required for the material to flow out of a recipient (V-funnel acc. to [4], ASTM C939 (2002), ISO 2431 $(02 / 1993))$. These methods are relatively simple but do not describe rheological properties in detail. The application of much more sophisticated methods is 
necessary to characterize the whole flow behavior. Rheometers controlling shear rate and measuring the resulting stress or vice versa are used to characterize the flow behavior of particle suspensions. Different measurement geometries (e.g., plate-plate, cylinder or others like the ball measurement system) are possible. Such methods mostly used for cement pastes also have been adapted for concrete; a comparison of concrete rheometers is given in $[5,6]$.

However, when adding fibers to concrete, mortar or paste, the determination of the rheological properties becomes difficult. The fibers may decrease the workability and lead to a much stiffer consistency by forming a kind of skeleton blocking the free flow of the cement matrix. Hence only a part of the material is sheared as the yield stress is not exceeded homogeneously. Additionally the fibers may accumulate during measurement and act as local disturbance.

The rheological characterization of the fiber reinforced composites is fundamental for the development of adequate mixture composition for many applications. As an example the development of a fiber reinforced composite for building elements like pillars, pylons and poles produced by a centrifugal casting method [7] is described. In the centrifugation technique a cylindrical mould is filled with the fresh mix, then closed and finally rotated around its symmetry axis. The conventional reinforcement of the concrete with steel bars is expensive and time consuming. The steel bars are susceptible to corrosion and hence rather thick and heavy structural elements result. The application of a fiber reinforced cementitious composite (FRC) or mortar is a suitable alternative. Besides extraordinary strength characteristics, a suitable FRC for this particular application also requires a very specific workability. The mixtures should not be too liquid to be able to fill the mould but not too stiff to be distributed evenly at the surface of the mould through the resulting centrifugal forces.

A blend of microfine cement and ordinary Portland cement improves the rheological properties of the fresh matrix and results in a very dense cement matrix with excellent mechanical properties [8]. This optimized cement matrix was reinforced with different kinds of carbon or polymeric fibers. The rheological parameters of the paste could be easily characterized by conventional methods. For the fiber reinforced composite this was not possible. A new method to characterize the fresh
Table 1 Chemical composition of the used cements

\begin{tabular}{lcc}
\hline & CEM I 42.5 N & microfine cement \\
\hline $\mathrm{CaO} /$ mass-\% & 62.8 & 45.3 \\
$\mathrm{MgO} /$ mass-\% & 2.0 & 8.1 \\
$\mathrm{SiO}_{2} /$ mass-\% & 19.9 & 32.6 \\
$\mathrm{Al}_{2} \mathrm{O}_{3} /$ mass-\% & 5.4 & 9.9 \\
$\mathrm{Fe}_{2} \mathrm{O}_{3} /$ mass-\% & 2.8 & 0.6 \\
$\mathrm{Na}_{2} \mathrm{O} /$ mass-\% & 0.13 & 0.3 \\
$\mathrm{~K}_{2} \mathrm{O} /$ mass-\% & 1.0 & 0.4 \\
$\mathrm{SO}_{3} /$ mass-\% & 2.8 & 2.6 \\
$\mathrm{~L} . \mathrm{O} . \mathrm{I} /$ mass-\% & 2.5 & 1.5 \\
\hline
\end{tabular}

consistency of fiber reinforced composites was developed for this purpose.

\section{Materials and methods}

Portland cement CEM I $42.5 \mathrm{~N}$ according to European Standard EN 197-1 and microfine cement were used as part of the binder system as described in [8-10]. The microfine cement had a medium grain size $\mathrm{d}_{50}=2.5$ $\mu \mathrm{m}$ and consisted to more than $80 \%$ of blastfurnace slag. The chemical compositions of the cements are given in Table 1.

For the measurement of paste rheology a rheometer (Paar Physica MCR 300) in controlled shear rate mode was used. It was equipped with a ball measuring system, developed especially for cement pastes and mortars [11-13]. It allows the measurement of suspensions up to a maximum grain size of about $4 \mathrm{~mm}$ and is also used in other fields of applications like debris flow material [14]. The measuring principle is shown in Fig. 1.

Instead of a laminar flow, as in other geometries like plate-plate or cylinder, a displacement flow is caused by an eccentrically rotating sphere. The sphere performs about $3 / 4$ of a full round with increasing rotation speed through the sample during data acquisition of the resulting torque.

According to [13] and also described in [14], the governing equations of the ball measurement system are as follows:

The movement of a sphere through a Newtonian fluid for Reynolds numbers $\mathrm{Re}<1$ can be expressed as:

$\mathrm{F}=3 \cdot \pi \cdot \eta \cdot \mathrm{v} \cdot \mathrm{D}$ 
Fig. 1 Principle of a rheometer with a sphere measuring system according to Tyrach [13] (scheme adapted from [14]).

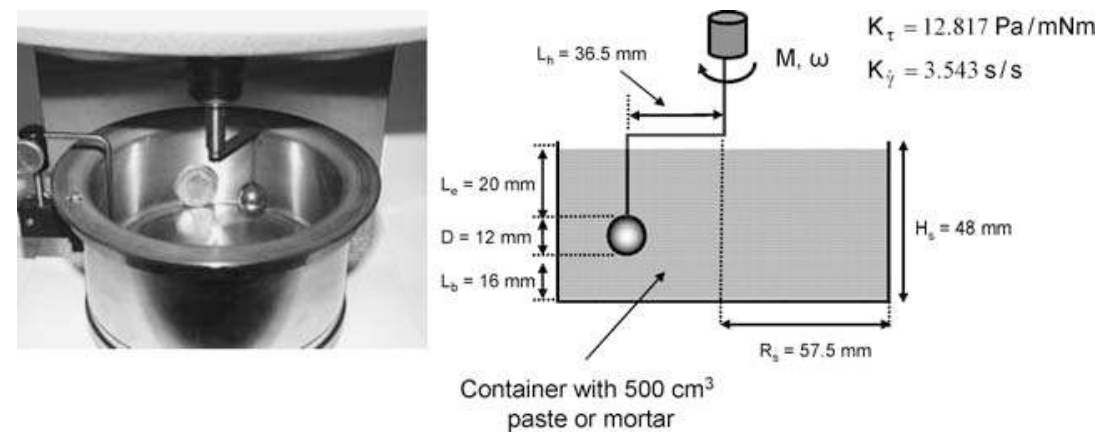

where

F: force

$\eta$ : dynamic viscosity of the fluid

$\mathrm{v}$ : velocity of the sphere

D: diameter of the sphere

Concerning non Newtonian fluids like particle suspensions the assumption had been made that the Stokes equation is also valid in that case.

The Reynolds number is defined as:

$\operatorname{Re}=\frac{\mathrm{v} \cdot \mathrm{D} \cdot \rho}{\eta}<1$

where

Re: Reynolds number

$\rho$ : fluid density

Torque and sphere velocity are given as:

$\mathrm{M}=\mathrm{F} \cdot \mathrm{L}_{\mathrm{h}}$

$\mathrm{v}=\omega \cdot \mathrm{L}_{\mathrm{h}}$

where

M: torque

$\mathrm{L}_{\mathrm{h}}$ : distance between sphere and container center

$\omega$ : angular velocity

From equations (1), (3) and (6) the torque $M_{1}$ is given as:

$\mathrm{M}_{1}=3 \cdot \pi \cdot \eta \cdot \omega \cdot \mathrm{D} \cdot \mathrm{L}_{\mathrm{h}}^{2}$

An additional torque $\mathrm{M}_{2}$ results from self-rotation of the sphere:

$\mathbf{M}_{2}=\pi \cdot \eta \cdot \omega \cdot \mathrm{D}^{3}$
The total torque $\mathrm{M}$ is

$\mathrm{M}=\mathrm{M}_{1}+\mathrm{M}_{2}=\pi \cdot \eta \cdot \omega \cdot\left(3 \cdot \mathrm{L}_{\mathrm{h}}^{2} \cdot \mathrm{D}+\mathrm{D}^{3}\right)$

Dynamic viscosity is given as

$\eta=\frac{\tau}{\dot{\gamma}}$

where

$\tau: \quad$ shear stress

$\dot{\gamma}$ : shear rate

From equations (7) and (8) the linear relationships for shear rate and shear stress can be derived.

$\dot{\gamma}=\frac{\pi \cdot \tau}{\mathrm{M}} \cdot\left(3 \cdot \mathrm{D} \cdot \mathrm{L}_{\mathrm{h}}^{2}+\mathrm{D}^{3}\right) \cdot \omega=\mathrm{K}_{\dot{\gamma}} \cdot \omega$

$\tau=\frac{\dot{\gamma}}{\pi \cdot \omega} \cdot \frac{1}{3 \cdot \mathrm{D} \cdot \mathrm{L}_{\mathrm{h}}^{2}+\mathrm{D}^{3}} \cdot \mathrm{M}=\mathrm{K}_{\tau} \cdot \omega$

This linear relationship was verified by [13] using different polymer solutions. The proportional factors are related to the geometry of the system (influence of the thin ball holder, container wall and bottom etc.). Their values for the applied geometry are given in Fig. 1.

The ball measurement system has several advantages compared to conventional systems (e.g., cylinder or plate-plate), when used for building materials: wall slip and shear induced sedimentation are minimized. However it has to be kept in mind that the measuring time at high shear rates is quite short so that a steady state of flow might not be reached.

Pastes with a water/binder ratio of 0.20 were prepared using an EN 196-1 type mortar mixer. 2 mass-\% of binder of a polycarboxylate type superplasticizer (solid content $32 \%$ ) were added. The time of mixing 
Table 2 Fiber properties

\begin{tabular}{lll}
\hline & Carbon $\left(\right.$ PAN $\left.^{*}\right)$ & PVA \\
\hline Young's modulus & $228 \mathrm{GPa}$ & $40 \mathrm{GPa}$ \\
Tensile strength & $3.8 \mathrm{Gpa}$ & $1.6 \mathrm{GPa}$ \\
Elongation at failure & $1.5 \%$ & $6.0 \%$ \\
Density & $1.81 \mathrm{~g} / \mathrm{cm}^{3}$ & $1.3 \mathrm{~g} / \mathrm{cm}^{3}$ \\
Diameter & $7.2 \mu \mathrm{m}$ & $40 \mu \mathrm{m}$ \\
Length & $6.4 \mathrm{~mm}$ & $6 \mathrm{~mm}$ \\
& & $12 \mathrm{~mm}$ \\
\hline
\end{tabular}

*based on polyacrylonitrile.

was 3 min at $62.5 \mathrm{rpm}$ according to EN 196-3. Immediately after mixing, a flow curve with shear rates from $0.01 \mathrm{~s}^{-1}$ to $100 \mathrm{~s}^{-1}$ was recorded. Apparent yield stress and plastic viscosity were calculated according to the Bingham model.

When fibers (see Table 2 for the properties of the used fibers) are added to the above described cement paste, the determination of the rheological parameters with this method gets difficult. The same applies for other rheological methods. Hence, for the evaluation of the rheological properties of the fiber reinforced composites, a new method has to be developed. It is based on an active squeezing of the fresh material out of a vessel. The measurement arrangement is shown in Fig. 2. A conical measuring bob is moved at a constant velocity into a hollow cone filled with the fresh mixture. The resulting force is detected in function of the displacement. Alternatively, the force may be kept constant and the displacement is measured, which could not be applied in this test setup. In this way, even very stiff and high volume fiber composites can be analyzed.

The vessel allows an easy and well reproducible filling. The volume of the vessel and the opening angle was chosen so that a representative material volume could be measured. If necessary, the geometrical size may be adapted in order to measure mixtures with larger maximum grain size or longer fibers. The opening angle of $60^{\circ}$ is a compromise between high filling volume, a steady flow and the overall dimensions of the vessel.

In this test arrangement the material is flowing between two nearly parallel walls. Owing to the very stiff consistencies of the studied mixtures, plug flow is expected. An orientation of the fibers is unlikely. This is a big advantage in comparison with an evaluation of rheological parameters in an extrusion test arrangement [15]. The gap width between these walls decreases starting from $50 \mathrm{~mm}$ to $5 \mathrm{~mm}$ when the mea-
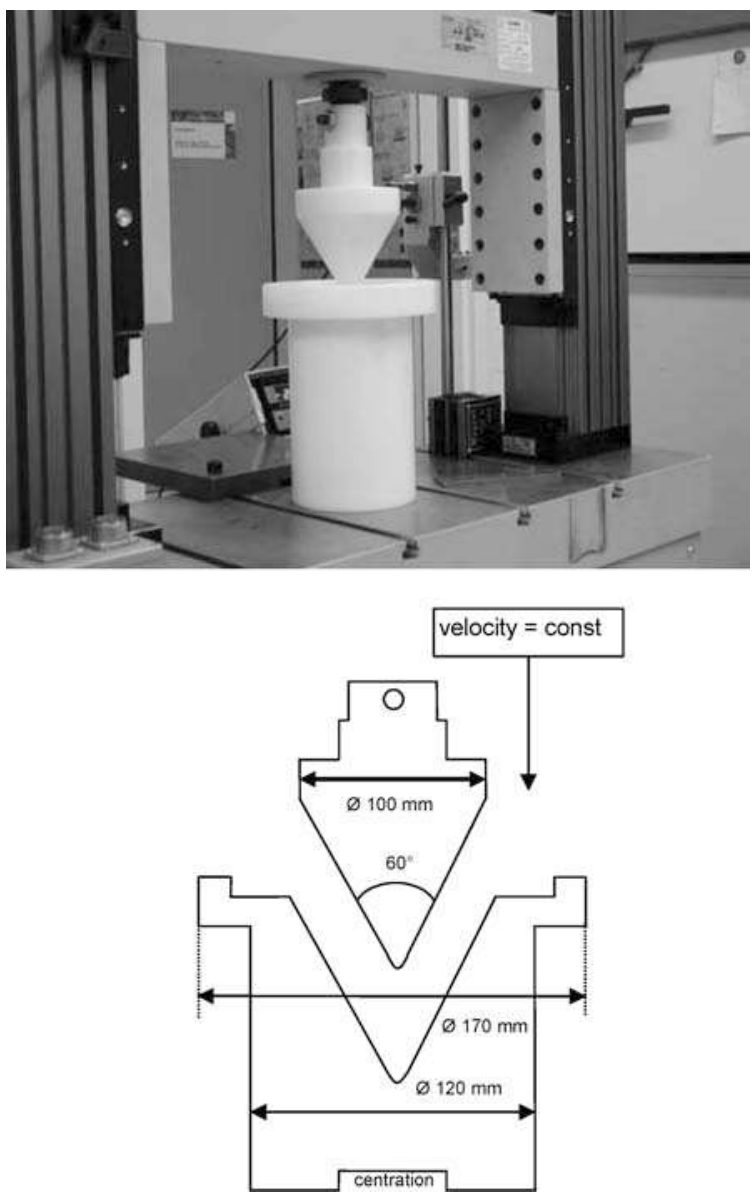

Fig. 2 Test arrangement for the evaluation of the coneconsistency.

suring bob is driven down. This increases the shear stress and hence the observed force during the experiment. Both the yield value and the plastic viscosity of a material influence the result. The recorded force is an integral of these parameters. A direct correlation to absolute rheological parameters however is not possible because of the complex flow geometry in the vessel.

Figure 3 shows a typical result. The measured force is plotted in function of the displacement of the measuring bob. The more the force curve is shifted towards higher displacement, the better is the workability of the mixture.

The velocity of the crosshead influences the result. The faster the crosshead is lowered, the higher is the resulting flow velocity of the material between the vessel and the crosshead. For Newtonian or Bingham viscous material this would lead to lower forces at the same displacement of the measuring bob because of higher 


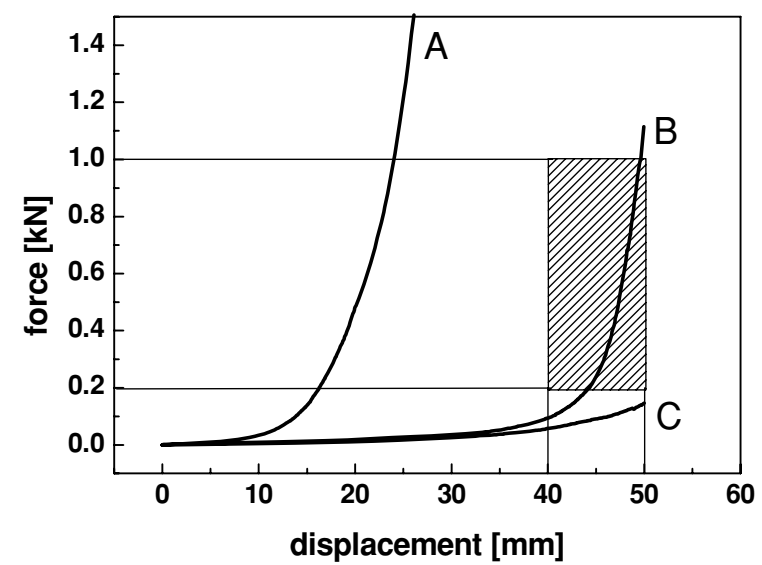

Fig. 3 Typical result from cone-consistency measurement and qualification of consistency for centrifugation casting.

shear tension. In the case of thixotropic or structure viscous material, the opposite may be the case, because the inner structure of the material would be broken by the faster movement. This later case actually was observed with the highly packed microfine cement blend (Fig. 4). This thixotropic character is ideal for a spin casting process. For standard tests a speed velocity of $0.5 \mathrm{~mm} / \mathrm{min}$ was chosen. This velocity lead to an optimal usage of the measurement range (displacement and force).

The described method was applied to evaluate the suitability of fiber reinforced composites for an application in centrifugal casting technique $[16,17]$. This is very important as prototyping is very time and material consuming. Comparing the homogeneity of the wall thickness of centrifuged prototypes (at centrifugal

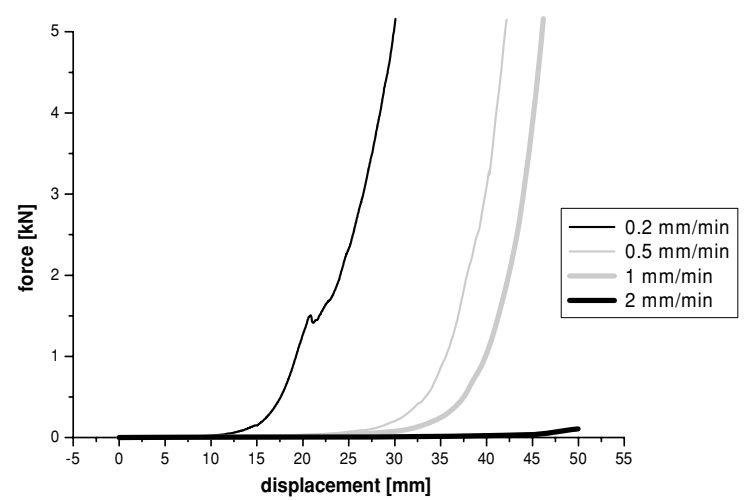

Fig. 4 Influence of the velocity of the measuring bob on the cone-consistency (cement/microfine cement $=80: 20 ; 1.0$ vol.$\%$ carbon fiber). acceleration higher than $15 \mathrm{~g}$ at smallest diameter) with the cone-consistency, a criteria for good centrifugability was defined: The force-displacement must cross the region between 0.2 and $1 \mathrm{kN}$ at a displacement between 40 and $50 \mathrm{~mm}$ (see Fig. 3, curve B and Fig. 5B). This criterion was found comparing the consistency curves with spun cast pipes of more than 20 different mixtures. When the force-displacement curve misses this region to the left side (see curve A in Fig. 3) the consistency is too stiff, and no homogenous element is formed (see Fig. 5A). Curve $C$ in Fig. 3 shows a mixture that is too liquid which may result in disintegration of the centrifuged element due to a low green body strength (see Fig. 5C), segregation or mould filling problems (the mould generally has to be overfilled to reach critical wall thickness).

The criteria for a good workability in the centrifugal casting, depends to a certain extent on the centrifugation speed (e.g., the centrifugal acceleration). At high speed somewhat stiffer consistencies may be used. The force limit at $50 \mathrm{~mm}$ however should be left untouched.

To evaluate the reproducibility of the coneconsistency test, the same mixture was prepared six times. The force-displacement curves of six individual measurements are plotted in Fig. 6. The reproducibility is very good. This mix was quite stiff, so that slump flow could not be derived.

\section{Results}

The above described cone-consistency method is applied to a fiber reinforced composite based on a cementitious matrix containing microfine cement. The rheological parameters (rheometer with ball measurement system) of the pastes without fibers are plotted in Fig. 7. The addition of microfine cement lowers yield stress and plastic viscosity significantly. An optimum is reached at 20 mass- $\%$ of microfine cement. The reduction is similar as with inert limestone filler (given as comparison) and much stronger compared to microsilica [18].

The mixture 80 mass- $\%$ Portland cement, $20 \%$ microfine cement and 2 mass- $\%$ of superplasticizer referred to binder serves as a standard matrix for the following studies. When adding carbon fibers, the consistency changes significantly so that it cannot be measured using conventional rheological measurements. It is hence studied with the cone-consistency test setup. 

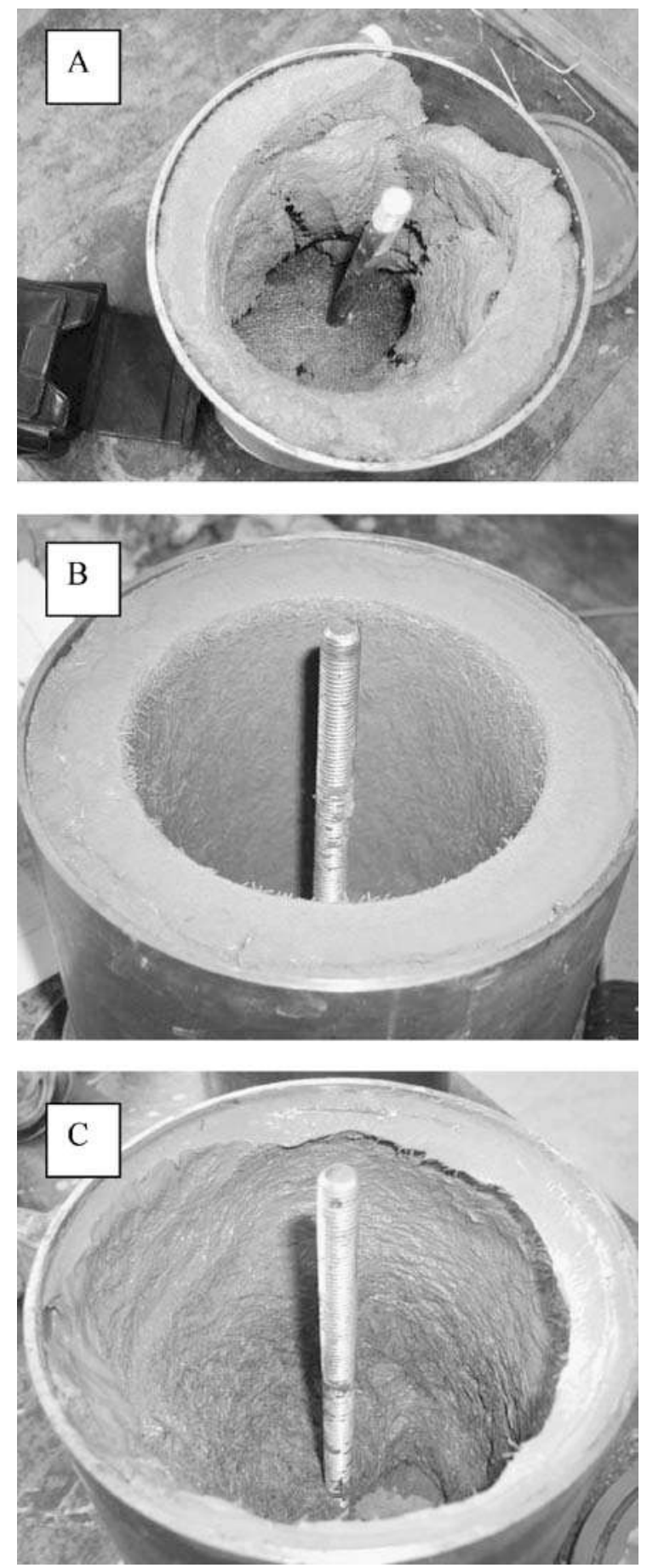

Fig. 5 (A) Appearance of a very stiff mixture after spinning (inhomogeneous wall thickness). (B) Homogeneous wall thickness after spinning. (C) Appearance of a very liquid mixture after spinning (low green body strength, segregation, inhomogeneous wall thickness).

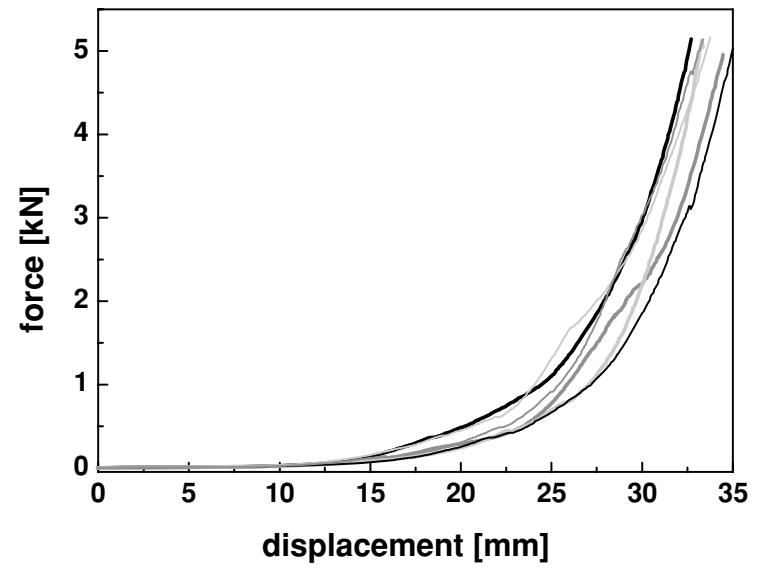

Fig. 6 Reproducibility of cone-consistency: 6 times the same mixture (cement $/$ microfine cement $=80: 20 ; 1.8$ vol.- $\%$ carbon fiber).

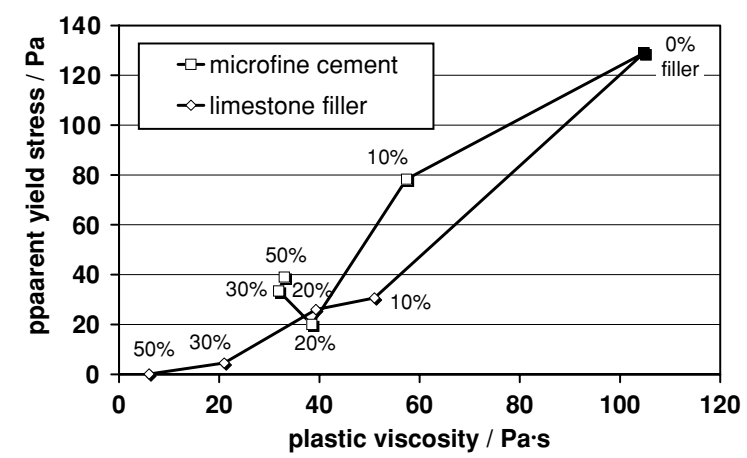

Fig. 7 Bingham parameters of the investigated cement pastes $(\mathrm{w} / \mathrm{b}=0.20)$ with different filler contents ( $2 \%$ superplasticizer).

The influence of the water/binder ratio on a 1 vol.- $\%$ (standard matrix) carbon fiber reinforced composite is shown in Fig. 8. As expected, a higher w/b ratio leads to a better fluidity. The $\mathrm{w} / \mathrm{b}=0.18$ mixture is already too fluid, missing the criteria for good centrifugal casting. Increasing the carbon fiber content leads to very stiff mixtures hardly fulfilling the criteria (see Fig. 9). An increase in fiber volume of only 0.25 vol.- $\%$ changes a too liquid mixture to an almost too stiff one. The reason for this is the very high aspect ratio and the large surface of the used carbon fibers. Thicker fibers certainly would improve workability, but production of thicker (PAN-) based fibers increases the price of this fiber type to a large extent.

A further possibility to increase workability of carbon fiber reinforced composites is the addition of polymer dispersions to improve wetting properties of the 


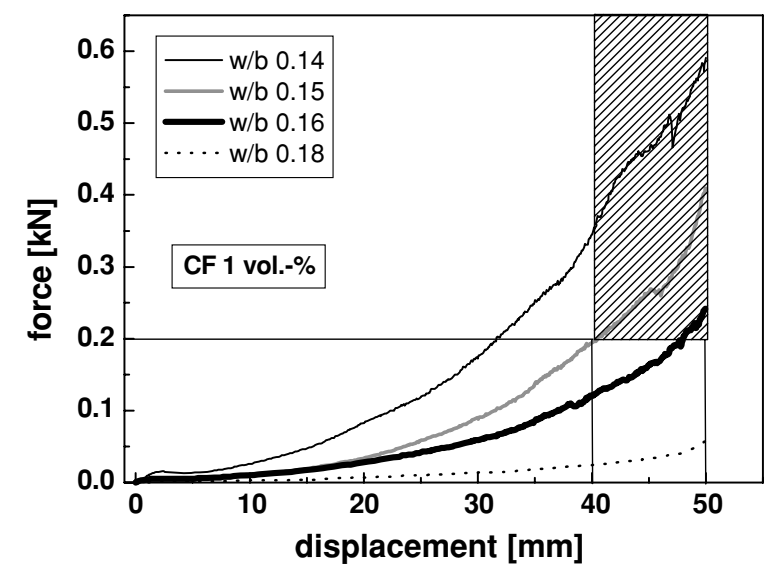

Fig. 8 Influence of water/binder ratio on the cone-consistency of mixtures with low carbon fiber contents (1 vol.-\%).

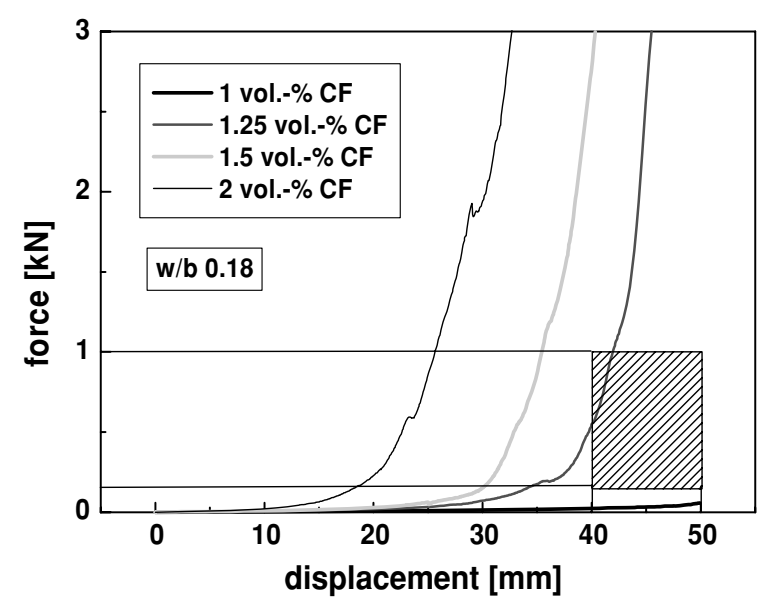

Fig. 9 Influence of carbon fiber content on the cone-consistency of mixtures with a high fiber content.

fiber in the fresh cementitious matrix. Different polymer types (properties see Table 3 ) were tested. Their effect at a dosage of 3 mass- $\%$ on the cone-consistency of a 2 vol.- $\%$ carbon fiber reinforced composite (standard

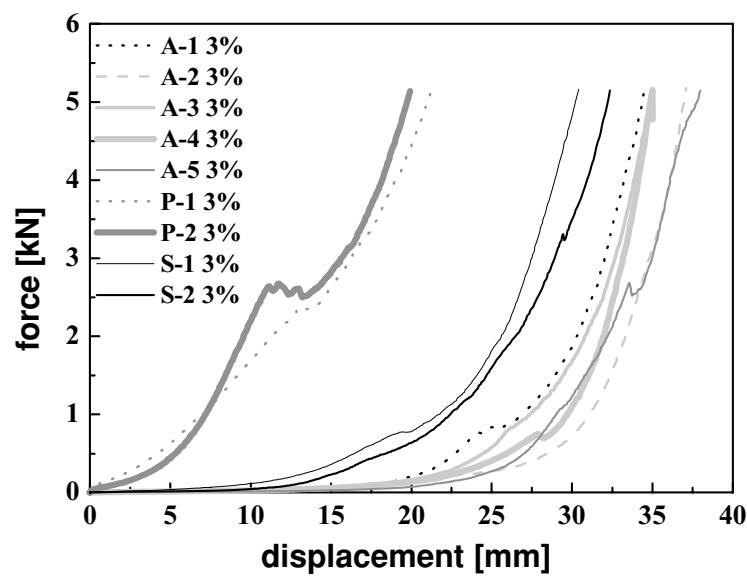

Fig. 10 Influence of the different polymer dispersions on the cone-consistency of carbon fiber-composites (CEM I $42.5 \mathrm{~N}$ $80 \%$, microfine cement $20 \%$, superplasticizer $3 \%$, w $/ \mathrm{b}=0.20$, carbon fibers 2 vol.- $\%$ ).

matrix) is shown in Fig. 10. Good results are obtained with polymers containing acrylic groups. Figure 11 shows the effect of the dosage of polymer S-2 (latex suspension). A low polymer content does not improve the cone-consistency very much, whereas the workability increases significantly with higher amounts.

Besides workability, strength performance is important. Concerning polymer dispersions it depends on the film forming ability that depends on the film forming temperature.

The influence of the polymer S-2 with a low film formation temperature on the strength properties of a 2 vol.-\% carbon fiber reinforced composite (3 point bending, span $80 \mathrm{~mm}$, specimens $25 \mathrm{~mm} \times 25 \mathrm{~mm} \times$ $100 \mathrm{~mm}$ ) is shown in Fig. 12. The bending strength increases with increasing polymer dosages. An optimum is found at an additive content of $2-3$ mass- $\%$. At

Table 3 Properties of the studied polymer admixtures

\begin{tabular}{|c|c|c|c|c|c|}
\hline & Composition & $\begin{array}{l}\text { Viscosity } \\
\mathrm{mPa} \cdot \mathrm{s}\end{array}$ & $\begin{array}{l}\text { film formation } \\
\text { temperature }{ }^{\circ} \mathrm{C}\end{array}$ & $\begin{array}{l}\text { glass formation } \\
\text { temperature }{ }^{\circ} \mathrm{C}\end{array}$ & $\begin{array}{l}\text { particle } \\
\text { size } \mu \mathrm{m}\end{array}$ \\
\hline A-1 & Copolymer of acrylonitrile and acrylic acid ester & $30-50$ & 50 & 70 & 0.1 \\
\hline A-2 & Copolymer of acrylonitrile and methacrylic acid & $500-1300$ & 13 & 20 & 0.1 \\
\hline A-3 & Copolymer of acrylic acid ester & $5-35$ & 55 & 72 & - \\
\hline A-4 & Copolymer of acrylic acid ester and styrene & $140-200$ & $<1$ & -6 & 0.2 \\
\hline A-5 & Copolymer of acrylic acid ester and styrene & $140-200$ & $<1$ & -6 & 0.2 \\
\hline P-1 & Polyacrylate, ammonium salt & $20-50$ & - & - & - \\
\hline P-2 & Polyacrylate, sodium salt & $80-250$ & - & - & - \\
\hline S-1 & Dispersion based on styrene and butadiene & $100-300$ & 25 & - & 0.2 \\
\hline $\mathrm{S}-2$ & Dispersion based on styrene and butadiene & $35-150$ & 16 & - & 0.2 \\
\hline
\end{tabular}




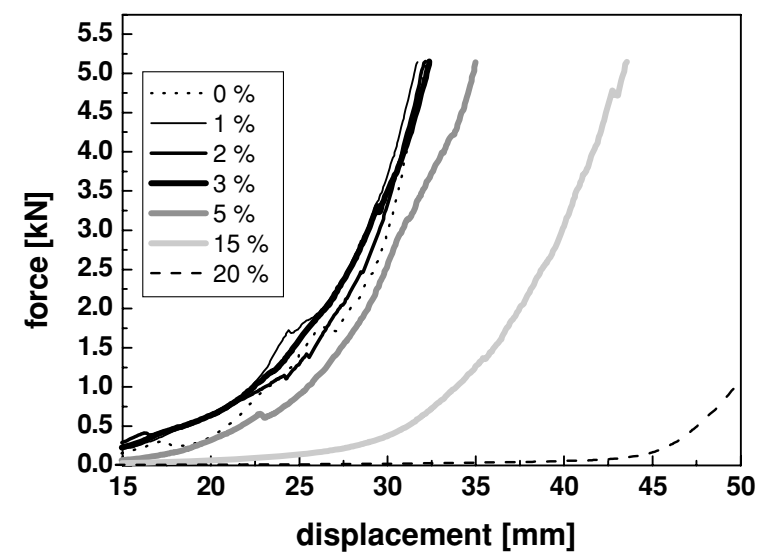

Fig. 11 Effect of amount of polymer (S-2) on the cone-

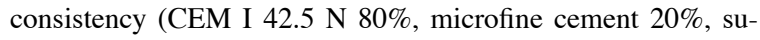
perplasticizer $3 \%$, w $/ \mathrm{b}=0.20$, carbon fibers 2 vol.- $\%$ ).

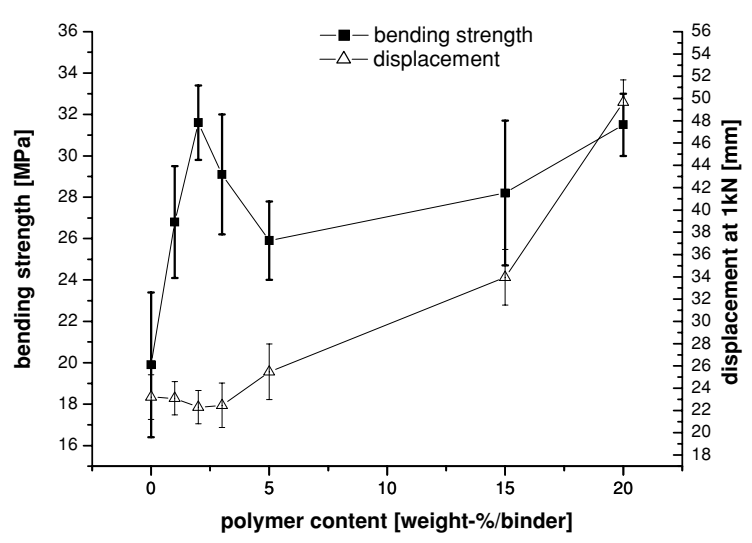

Fig. 12 Influence of the amount of polymer dispersion (S-2) on the cone-consistency and the flexural strength of CF-composites

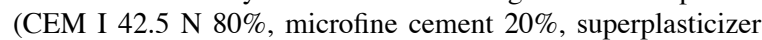
$3 \%, \mathrm{w} / \mathrm{b}=0.20$, carbon fibers 2 vol.- $\%$ ).

higher dosages the strength first decreases slightly. At dosages higher than $15 \%$ a further strength gain is observed. An explication for this behavior may be given as follows: At lower content the polymer suspension mayncrease workability and improve, owing to film formation, the matrix-fiber bond. At higher content the polymer could disturb the hydration process of the matrix by confinement of the cement particles. This leads to a strength loss despite a better workability. At much higher dosages, the polymer itself is responsible for the strength development and replaces partly the cement paste and a polymer bonded composite results. However, both strength and workability can be improved with high polymer contents, but the economical considerations may favor the optimum at medium polymer

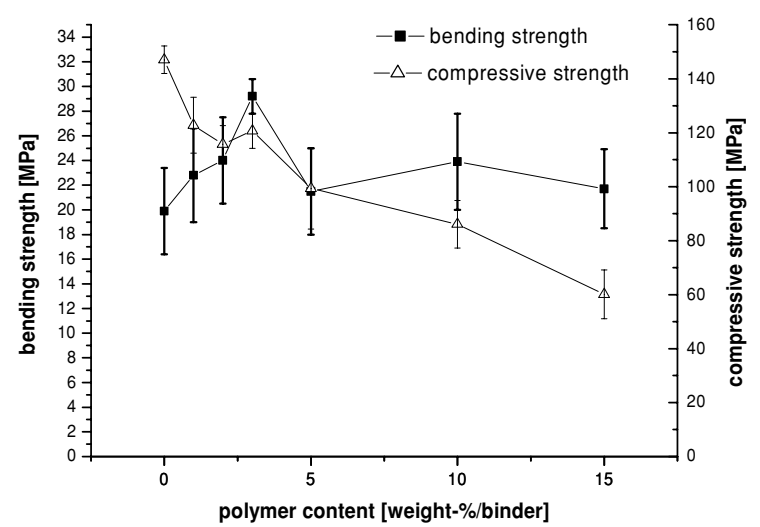

Fig. 13 Effect of the amount of polymer on the compressive strength of hardened composites (carbon fibers 2 vol.-\%, A-3, high film formation temperature).

contents. The effect of a higher film formation temperature on carbon fiber reinforced composites is shown with polymer A-3 and plotted in Fig. 13. While bending strength is also found to be slightly increased for medium polymer content, higher contents do not lead to an improvement. The compressive strength shows a mostly negative effect of the polymer addition owing to hindrance of cement hydration.

The fiber selection plays a crucial role concerning the workability of fiber reinforced composites. Besides surface properties (material, sizing), the geometry (e.g., surface area) is the decisive parameter. Hence using a coarser fiber with higher affinity to cementitious suspensions leads to a much better workability as is shown in Fig. 14. Up to 5 vol.-\% of a PVA fiber may be used

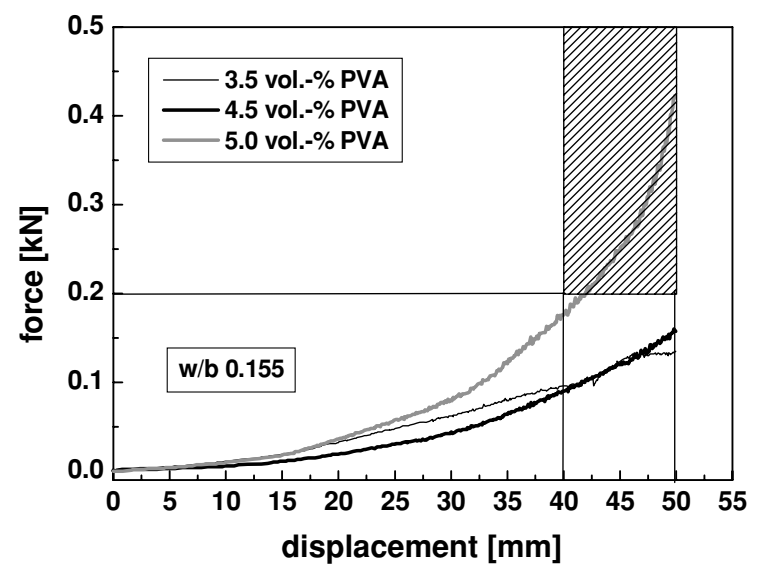

Fig. 14 Influence of PVA-fiber content on the cone-consistency (75\% CEM I $42.5 \mathrm{~N}, 25 \%$ microfine cement, superplasticiser $2.5 \%)$. 
without missing the criteria for a good centrifugal casting ability at very low $\mathrm{w} / \mathrm{b}$ ratio with the standard matrix containing microfine cement.

\section{Conclusions}

The workability of fiber reinforced cementitious composites, especially when used in the spun casting process, is difficult to determine by conventional testing methods like the flow table test or by rheological measurements. However, there is a strong need for the development of a consistency testing methods for this kind of systems, because on-site application tests are time consuming and expensive.

To solve this problem, a special testing setupthe cone consistency test-was developed. A conical measuring bob is moved with constant velocity into a hollow cone containing the freshly mixed composite. The resulting force is recorded as function of the displacement.

Comparing the obtained data with data from on-site testing a criterion for mixtures suitable for the spun casting process was developed. The reproducibility of the cone consistency test revealed to be quite good, so that the method can be used to optimize fiber reinforced composites.

Using the cone consistency test, the influence of different fibers (type, dimensions) and of different polymers on workability properties of the composites with respect to the spun casting process could be characterized.

The results show that the cone consistency test is a suitable method to characterize the workability properties of fiber reinforced cementitious composites. The number of time consuming and expensive on-site application tests can be reduced.

This method was applied to spin casting. In addition, it might be useful for any other fabrication process that requires stiff or high fiber volume mixtures, among them compaction casting (pipes, thin cement boards, pre-fabricated elements and other products), extrusion [19] and pulltrusion processes [20]. Even earth-moist cementituous mixtures might be characterized and their rheological properties under pressure could be studied with the described method.

Acknowledgements This paper presents some results of a threeyear research project on the application of fiber reinforced high performance composites in spun-casting fabrication. The project was supported by the Swiss Commission for Technology and Innovation (KTI/CTI) and SACAC AG (Lenzburg, Switzerland).

\section{References}

1. Tattersall GH, Banfill PFG (1983) The rheology of fresh concrete. Pitman Books Limited, London.

2. Banfill P, (ed.) (1991) Rheology of fresh cement and concrete. E. \& F.N. Spon, London.

3. Ferraris CF (1999) Measurement of the rheological properties of high performance concrete: state of the art report. Journal of Research of the National Institute of Standards and Technology 104: 461-478.

4. Nawa T, Izumi T, Edamatsu Y (1998) State-of-the-art report on materials and design of self-compacting concrete. Int. Workshop on Self-Compacting Concrete, Kochi, Japan, August, 160-190.

5. Banfill PFG, Beaupré D, Chapdelaine F, de Larrard F, Domone P, Nachbaur L, Sedran T, Wallevik O, Wallevik JE (Sept. 2001) Comparison of concrete rheometers: International tests at LCPC (Nantes, France) in October 2000, Report NISTIR 6819, National Institute of Standards and Technology, Building and Fire Research Laboratory, ed. Ferraris $\mathrm{CF}$, Brower LE.

6. Ferraris CF, Martys N (2003) Relating fresh concrete viscosity measurements from different rheometers. Journal of Research of the National Institute of Standards and Technology 108:229-234.

7. Dilger WH, Ghali A, Rao S (1996) Improving the durability and performance of spun-cast concrete poles. PCI Journal, 41(2):68-90.

8. Kaufmann J, Winnefeld F (2002) Influence of addition of ultrafine cement on the rheological properties and strength of Portland cement paste. Proc. Int. Conf. Innovations and Developments in Concrete Materials and Construction, Dundee, UK 827-836.

9. Kaufmann J, Matschei Th, Hesselbarth D (2003) Effect of the addition of ultrafine cement on the properties of fiber reinforced composites. Proc. 11th Int. Conference on the Chemistry of Cement ICCC Conference, Durban South Africa 1683-1691.

10. Kaufmann J, Winnefeld F, Hesselbarth D (2004) Effect of the addition of ultrafine cement and short fiber reinforcement on shrinkage, rheological and mechanical properties of Portland cement pastes. Cement and Concrete Composites 26:541549.

11. Physica Messtechnik GmbH (1999) A new ball measuring system for large particle suspensions. Applied Rheology 9:145-146.

12. Müller M, Tyrach J, Brunn PO (1999) Rheological characterization of machine-applied plasters. ZKG International 52:252-258.

13. Tyrach J (2001) Rheological characterization of cementitious building materials. $\mathrm{PhD}$ thesis, University of ErlangenNürnberg, 2001 (only available in German).

14. Schatzmann M, Fischer P, Bezzola GR. Rheological behavior of fine and large particle suspensions. Journal of Hydraulic Engineering-ASCE 129(10):796-803. 
15. Zhou X, Li Z (2005) Characterization of rheology of fresh fiber reinforced cementitious composites through ram extrusion. Mater. Struct., 38:17-24.

16. Hesselbarth D, Kaufmann J (2004) The role of fine supplementary binder in high performance fiber reinforced cementitious composites, 8th Canmet/ACI Int. Conf., SP-221 on fly ash, silica fume, slag and natural pozzolans in concrete, Las Vegas, pp. 395-402.

17. Kaufmann J, Hesselbarth D, Moser K, Terrasi GP (2005) Application of fiber reinforced high performance composites in spun-cast elements. Materials and Structures 38:549-555.
18. Nehdi M, Mindess S, Aïtcen P-C (1998) Rheology of high-performance concrete: effect of ultrafine particles. Cement and Concrete Research 28:687697.

19. Peled A, Akkaya Y, Shah SP (2000) Parameters related to fiber length and processing in cementitous composites. Mater. Struct., 33:515-524.

20. Peled A, Bentur A, Mobasher B (2004) Pulltrusion versus casting processes for the production of fabric-cement composites. Proc. Sixth int. RILEM Symp. BEFIB, pp. 14951504. 